\title{
Capítulo 6 Biosíntesis de Nano Partículas Metálicas
}

\section{Chapter 6 Biosynthesis of Nano Metallic Particles}

LOZANO-CAMARGO, Maria Luisa†*, LORENZILLA-GARCÍA, Jatziri, GALICIA-LUIS, Laura y BARRERA-CALVA, Enrique

Tecnológico de Estudios Superiores del Oriente del Estado de México Universidad Autónoma Metropolitana-Iztapalapa

ID $1^{\mathrm{er}}$ Autor: Maria Luisa Lozano-Camargo/ORD ID: 0000-0002-0777-6392, CVU CONACYT ID: 46638

ID $1^{\text {er }}$ Coautor: Jatziri Lorenzilla-García/ ORD ID: 0000-0001-5406-872X

ID $2^{\text {do }}$ Coautor: Laura, Galicia-Luis / CVU CONACYT ID: 120121

ID $3^{\text {er }}$ Coautor: Enrique, Barrera Calva/ ORD ID: 0000-0002-3041-5552

DOI: $10.35429 /$ H.2020.8.69.85

M. Lozano, J. Lorenzilla, L. Galicia y E. Barrera

maria.lozano@tesoem.edu.mx

A. Marroquín, J. Olivares, L. Cruz y A. Bautista. (Coord) Ciencias ambientales, uso de recursos. Handbooks-CECORFANMexico, Querétaro, 2020. 


\title{
Resumen
}

En las últimas décadas se han incrementado las investigaciones sobre nuevos métodos de síntesis de nanopartículas metálicas, que sean capaces de cubrir las demandas actuales de energía, economía y medio ambiente. a síntesis de nanopartículas de óxidos de manganeso (MnO), los cuales son favorables para su uso en "capacitores eléctricos", dadas sus particularidades ópticas, físicas y químicas, así como su gran capacidad para almacenar grandes cantidades de energía de ahí el nombre de "capacitores". En este trabajo se empleó un método de síntesis verde de nanopartículas de óxidos de zinc (ZnO) y óxidos de manganeso (MnO) presentes en los residuos de las pilas zinc-carbón, empleando la biomasa del Lirio Acuático (Eichhoirnia Crassipies) como agente biorreductor, se seleccionó el lirio por las características que esta planta presenta en la acumulación de metales y su empleo para eliminar metales pesados en las plantas de tratamiento de agua, los nanometales obtenidos, fueron analizados mediante la técnicas de difracción de Rayos X (DRX) y microanálisis por energía dispersiva de rayos X (EDX) con el Microscopio de Transmisión de Electrones (TEM por sus siglas en inglés); que también permite micrografías para identificar su estructura y tamaño obtenido.

\section{Síntesis Nano partículas, óxidos de manganeso, oxido de zinc, pilas zinc-carbón, lirio acuático}

\begin{abstract}
In recent decades, research has increased on new methods of synthesis of metallic nanoparticles, which are capable of meeting current energy, economic and environmental demands. The synthesis of nanoparticles of manganese oxides (MnO), which are favorable for use in "electrical capacitors", given their optical, physical and chemical characteristics, as well as their great capacity to store large amounts of energy, hence the name of "Capacitors". In this work, a green synthesis method of nanoparticles of zinc oxides $(\mathrm{ZnO})$ and manganese oxides $(\mathrm{MnO})$ present in the residues of zinc-carbon cells was used, by means using the biomass of the Water Lily (Eichhoirnia Crassipies) as a bioreducing agent, the lily was selected for the characteristics that this plant presents in the accumulation of metals and its use to eliminate heavy metals in water treatment plants, the obtained nanometals were analyzed by means of $\mathrm{X}$-ray diffraction, DRX, and X-ray energy dispersive spectroscopic (EDX) microscope techniques. Transmission System (TEM) to identify its structure and size was also used.
\end{abstract}

\section{Synthesis Nano particles, manganese oxides, zinc oxide, zinc-carbon batteries, water lily}

\section{Introducción}

En los últimos años los nanomateriales han sido de gran interés para la comunidad científica-tecnológica por presentar propiedades físicas, químicas, electrónicas y magnéticas diferentes a sus estructuras macroscópicas; sus dimensiones se encuentran en un rango de la nanoescala (inferior a 100 nanómetros), cuentan con una mayor área superficial, haciendo más activo a éstos materiales, sus efectos cuánticos pueden producir cambios significativos en sus propiedades en comparación con sus análogos en volumen. En esta escala, los cambios cualitativos en las propiedades fisicoquímicas y la reactividad están ligadas al número de átomos o moléculas que forman el material, permitiendo observar el efecto del tamaño en fenómenos tales como: la resonancia de plasmón (es un cuanto de oscilación del plasma; es decir, la cuasipartícula que resulta de las oscilaciones del plasma) superficial (RPS) en las nanopartículas metálicas, el confinamiento cuántico en partículas semiconductoras y el superparamagnetismo en nanomateriales magnéticos (Roudner, 2006; Jun, et col., 2007); En el siglo XIX Faraday realizó trabajos de síntesis y estudios las propiedades ópticas de las nanopartículas (Faraday,1857).

En 1959 surge el concepto de nanociencia, en la conferencia de Richard Feynman titulada "There's a Plenty of Room at the Bottom" donde planteó la idea de una nueva ciencia basada en la manipulación individual de átomos y moléculas mediante un nuevo camino, abriendo paso al mundo de la multidisciplinariedad; no fue hasta 1974 que el término "nanotecnología" fue reconocido por Norio Taniguchi (Tokio Science University), como la separación, deformación de la materia a nivel atómico y molecular (N. Taniguchi, et. Col., 1974). Desde entonces se marca una nueva era en el avance científicotecnológico. 
Los científicos han desarrollado diversos estudios con la finalidad de establecer métodos seguros y reproducibles en la síntesis y fabricación de estos materiales controlando su tamaño, forma y estructura. La Agencia del Medioambiente de los EE.UU. clasifico a los nanomateriales basados en carbono en cuatro tipos los que presentan forma elipsoidal o esférica se conocen como fullerenos, los cilíndricos son nanotubos; los puntos cuánticos basados en metales de oro, plata y óxidos metálicos. La síntesis de nanopartículas se emplea con la finalidad de crear a nano escala estructurales de cadenas poliméricas largas como el ADN, para diseño de sensores automáticos que son empleados en diagnósticos y terapias de diversas enfermedades (cáncer, glucosa, etc.), así como para la fabricación de motores moleculares con polímeros inteligentes, biosensores y bio-sondas, etc.

Las nanopartículas se encuentran presentes en la vida cotidiana y procesos industriales, en su conjunto son esenciales en la nanociencia y la nanotecnología; los nanomateriales se han empleado de manera impresionante en el campo de la electrónica, óptica, la industria alimenticia, textil, cemento, plástico, y en la medicina Zarria y. Col. Realizaron una investigación de los efectos de las nanopartículas de $\mathrm{ZnO}, \mathrm{SiO}_{2}$ y $\mathrm{TiO}_{2}$ sobre la expresión génica de las interleuquinas 3 (IL-3) y 7 (IL-7) y el efecto estimulante de colonias de granulocito-macrófago (GMCSF), moléculas producidas en la MOR y otros grupos celulares. La síntesis de nanomateriales es empleada también para establecer métodos de biorremediación de desechos radiactivos (Uranio), en efectos antibacteriales y en la óptica no lineal. Estudios realizados con la magnetita y los materiales a base de silicio mostrando buenas propiedades eléctricas por lo que pueden ser usados en el sector energético, emplearon para ello, 5 ratones aplicándoles dosis que iban de 0.5 a $10 \mathrm{mg}$ de $\mathrm{SiO}_{2}$ y $\mathrm{ZnO}$ y de 5 a $25 \mathrm{mg}$ de $\mathrm{TiO}_{2}$ empleando como electrolito soporte una solución salina las cuales fueron inyectadas vía intraperitoneal, posteriormente fueron sacrificados para su extracción de medula ósea roja a partir de los huesos largos de patas anteriores y posteriores, logrando con ello, incrementar el conocimiento de los efectos perjudiciales de dichas partículas exponiendo a la médula ósea roja como un tejido adecuado para este tipo de evaluaciones y a los genes seleccionados como marcadores idóneos para la trazabilidad de estos efectos (Jacqulyne et. Col., 2017).

Serrano et. col. realizaron la optimización y síntesis de AUNPs empleando Hypericum perforatum y evaluando la actividad antimicrobiana combinando tratamientos biológicos y físicos, la extracción de Hypericum perforatum (HP) les permitió tener compuestos reductores y estabilizadores en la síntesis de partículas de oro a nanoescala, siendo una alternativa libre de materiales peligrosos o tóxicos, el emplear HP como biocatalizadores junto con el método por microondas es una ventaja ya que reduce la síntesis a 10 segundos en comparación con otros métodos biológicos (Serrano et. col, 2020).

Existe también diversos procedimientos que ofrecen grandes posibilidades para su aplicación a nivel industrial, al utilizar bacterias para la formación de cristales cuánticos semiconductores, al igual que inmovilizando sustancias bioactivas como enzimas, anticuerpos, ADN y ARN. (Perdigón, 2010). Una de las aplicaciones interesantes son las nanopartículas de óxido de hierro $(\mathrm{FeO})$ ya que presentan propiedades muy interesantes en nano-medicina por ser ampliamente biocompatibles, además de contar con propiedades magnéticas y eléctricas aplicables en la nanoelectrónica.

Las nanopartículas de $\mathrm{Ti} / \mathrm{Ni}$ presentan propiedades interesantes a escala nano, investigaciones realizadas por Schabes et col. indican que estás pueden ser empleadas como recubrimientos anticorrosivos, catalizadores y con una gran posibilidad utilizarse en materiales inteligentes o como memorias (P.S. Schabes et col., 2006); las nanopartículas de Pt/Pd tienen propiedades catalíticas muy eficientes (R. Herrera-Becerra, at col. 2008); así como las nanopartículas de Eu-Au son factibles para su empleo en el control nuclear, sensores ópticos, medicina nuclear y sobre todo en fotónica, las nanopartículas de $\mathrm{Yb}$ pueden emplearse en dispositivos ópticos y fotónicos logrando grandes avances en estos campos de la ciencia rompiendo así barreras preestablecidas (Ascencio et col. 2004); las nanopartículas de Sm presentan tamaños $\mathrm{nm}$ con buenos efectos cuánticos, momento magnético muy alto $\left(5 \mathrm{~m}_{\mathrm{B}}\right)$, colocando a estos materiales como nuevos magnetos permanentes de gran capacidad que los actuales, además de presentar propiedades catalíticas interesantes.

\subsection{Tipos de síntesis de nanometales}

Los métodos de síntesis de nanopartículas suelen agruparse en dos categorías:

- from top to bottom 
Consiste en dividir el sólido másico en proporciones más pequeñas, mediante la molienda o el desgaste, empleando métodos químicos o la volatilización de un sólido seguido por la condensación de los componentes volatilizados. (Baylon, 2015).

Existen varios métodos que utilizan la aproximación de <from top to bottom> los más utilizados son:

\section{a) La evaporación térmica}

Consiste en calentar el material que se pretende precipitar hasta que este comience a evaporarse, para lo cual se utiliza una cámara de vacío en la que se condensa el vapor sobre una lámina fría y se mantiene un control preciso de las condiciones de crecimiento para evitar modificaciones en la morfología de la capa depositada.

\section{b) Deposito químico en fase vapor}

Consiste en la descomposición de uno o varios compuestos volátiles, en el interior de una cámara de vacío (reactor), cerca de la superficie de un sólido para obtener la formación de un material en forma de capa delgada o de nanopartículas.

\section{c) Preparación de clusters gaseosos}

Emplea un láser de alta potencia que produce vapores atómicos metálicos los cuales son acarreados en un gas inerte y posteriormente son depositados en un óxido monocristalino u otro sustrato, bajo condiciones de ultra-alto vacío.

\section{d) Implantación de iones}

Consiste en la implementación de iones de un material en un sólido, modificando las propiedades físicas y químicas del sólido debido a que el ion implantado por ser un elemento distinto al que lo compone, causar cambios estructurales en el sólido implantado.

\section{e) Molienda de partículas de tamaño macro o micrómetro}

Se emplean molinos de alta eficiencia para obtener partículas de macro o micrométricas las cuales son clasificadas por métodos físicos, con la finalidad de recuperar las de tamaño micrométrico (Boldyrev, 2006).

\section{- bottom up}

Consiste en la fabricación de nanopartículas a través de la condensación de átomos o entidades moleculares en una fase gaseosa o en solución. Este último enfoque es mucho más utilizado. (Wachs, 2001).

Los métodos que más se emplean en la aproximación bottom up, son aquellos que utilizan procedimientos químicos. Los cuales inician con la reducción de los iones, metálicos a átomos metálicos, seguido por la agregación controlada de estos átomos. Ya que este método permite obtener nanopartículas uniformes y pequeñas. Los métodos más empleados son:

\section{a) Método coloidal}

Consiste en disolver una sal del precursor metálicos o del óxido a preparar, un reductor y un estabilizante en una fase continua o dispersante (un líquido). El agua puede emplearse como reductor, estabilizante o ambos. En principio el tamaño promedio, la distribución de tamaños y la forma o morfología de las nanopartículas se pueden controlar mediante variaciones en la concentración de los reactantes, del agente reductor y del estabilizante, así como la naturaleza del medio dispersante. 


\section{b) Reducción fotoquímica y radioquímica}

Es la síntesis de nanopartículas metálicas, se modifica el sistema químico mediante altas energías la cual está asociada con la generación de reductores fuertes altamente activos con electrones, radicales y especies excitadas. La reducción fotoquímica (fotolisis) y la radicación-química (radiolisis) difieren en el nivel de energía utilizado. La síntesis fotoquímica se caracteriza por energías por debajo de $60 \mathrm{eV}$, mientras que la radiolisis utiliza energías de 103-104 eV. Los métodos de reducción fotoquímica y radioquímica tienen como ventaja sobre el método de reducción química la ausencia de impurezas formadas cuando se usan agentes reductores químicos. Estos métodos producen nanopartículas de alta pureza. Además, la reducción fotoquímica y radioquímica permiten producir nanopartículas en condiciones de estado sólido y a bajas temperaturas. (Ershov, 1997).

\section{c) Irradiación con microondas}

La técnica de irradiación con microondas produce nanopartículas con una muy baja dispersión de tamaño, aunque no siempre se logre un control preciso en la morfología. Las microondas actúan como campos eléctricos de alta frecuencia, capaces de calentar cualquier material conteniendo cargas eléctricas como las moléculas polares en un disolvente o iones conductores en un sólido. Los solventes polares se calientan y sus componentes moleculares se ven obligados a girar con el campo y pierden energía en colisiones. Las muestras conductoras y semiconductoras se calientan cuando los iones y los electrones contenidos en ellas forman una corriente eléctrica y la energía se pierde debido a la resistencia eléctrica del material. (Zhu et al., 2004)

\section{d) Utilización de Dendrimeros}

La síntesis de nanopartículas también se ha llevado a cabo usando micelas, emulsiones y dendrimeros como nanorreactores que permiten la síntesis de partículas de forma y tamaños definidos. Esto se logra alterando la naturaleza de los dendrimeros. Los dendrimeros son moléculas altamente ramificadas, las que incluyen un núcleo central, unidades intermediarias repetitivas y grupos funcionales terminales (Muzafarov y Rebrov, 2000).

\section{e) Síntesis solvotermal}

En este se agrupan una serie de técnicas en las que un precursor metálico disuelto en un líquido, en recipiente cerrado, se calienta por encima de su punto de ebullición, lo que genera una presión superior a la atmosférica, el líquido habitual es agua, aunque cada vez se van utilizando con mayor frecuencia otros medios líquidos como: disolventes orgánicos, amoniaco líquido, hidracina, etc. Lo cual da a la síntesis solvotermal gran versatilidad. En este tipo de técnicas los tiempos de reacción son largos.

\section{f) Método sol-gel}

Es un proceso químico en fase húmeda ampliamente utilizado en la ciencia de los materiales. Este método se utiliza principalmente para la fabricación de nanomateriales. Se parte de una solución química o sol que actúa como precursor de una red integrada ya sea de partículas discretas o de una red de polímeros. Los precursores típicos son los alcóxidos y los cloruros metálicos, que sufren varias reacciones de hidrolisis y policondensación para formar una dispersión coloidal, que luego de una polimerización lenta forma un gel, precursor de los compuestos deseados. (Baylon, 2015)

\subsection{Biosíntesis de nanopartículas metálicas}

Hoy en día se han desarrollado diversos métodos para la síntesis de materiales a escala nanométrica con un control sobre la forma y tamaño de las nanopartículas suficientemente bueno para ser empleadas en diversas aplicaciones, sin embargo, la mayoría de los métodos de síntesis son costosos y agresivos con el medio ambiente (Frederix et, col., 2003)

En la búsqueda de tecnologías amigables con el medio ambiente se ha permitido el desarrollo de investigación dirigida hacia lo que hoy en día se define como "Síntesis verde o biosíntesis". El procedimiento de la biosíntesis de nanopartículas involucra la reacción del extracto vegetal con soluciones acuosas. (Baylón, 2015) 
Dicho método de biosíntesis o bio-reducción se reporta como una tecnología auto-sostenida, donde la biorremediación y la producción de partículas de metal están relacionadas. El uso de estos métodos o técnicas auto sostenibles abren las posibilidades de ser utilizado con otros elementos y plantas. (Yu et col., 2004)

El uso de los métodos de química verde cada vez es más importante, en particular, para la obtención de nuevos materiales a escala manométrica de metales y semiconductores, cabe señalar que en la actualidad es posible incluir el uso de compuestos de origen bilógico, como reductores y estabilizadores para la síntesis de nanoestructuras. Y se han reportado múltiples trabajos sobre la producción de pequeñas partículas de metal mediante el uso de bio-masa con ayuda de hongos, hierba de limón, geranio de tamarindo, de árbol de neem, entre otras plantas. (Yu et col., 2004).

En el caso específico de la síntesis de nanopartículas metálicas, se ha dirigido al uso de diferentes organismos como: bacterias o hongos, así como de plantas y sus extractos donde sus componentes participan como agentes biológicos en las reacciones de reducción y formación de nanopartículas metálicas. (Baylon, 2015).

El titanio un material de gran biocompatibilidad en los tejidos y huesos ya que permite el crecimiento de manera ordenada aun en contacto con las contingencias biofuncionales (infecciones por acumulación de placa bacteriana), estos implantes proporcionan propiedades antibacterianas y antifúngicas, la síntesis e inmovilización de nanopartículas de plata (AgNPs) sobre sustratos de titanio poroso con potencial osteointegrable por silanización, funcionalizando el titanio al adicionar moléculas orgánicas microrugosas, permite obtener substratos modificados superficialmente (Gaviria et. col., 2019).

Entre los principales metabolitos que tienen propiedades reductoras se encuentran los compuestos fenólicos como el ácido gálico, ácido benzoico y ácido cafeíco entre otros, que están presentes en algunas plantas. (Seung, Choi y J. Hwang, 2006).

Así por ejemplo se han obtenido nanopartículas de oro y plata a temperatura ambiente utilizando ácido gálico, un metabolito presente en algunos vegetales. (Karuvath, et col., 2007); también se ha llevado a cabo la síntesis de nanopartículas de oro por medio de un procedimiento sencillo empleando extracto de nopal (Opuntia ficus-indica) como agente reductor. (Baylón, 2015). La hoja de Té Negro, la cual es extremadamente eficiente en cuanto a biosíntesis debido a que lleva una rápida formación de nanopartículas estables de diferentes morfologías: esferas, trapezoides, prismas y varillas, utilizando tres diferentes soluciones acuosas de extractos derivados de las hojas del Te Negro para la síntesis de oro y plata. (Ara et al., 2009).

Plantas como la de alfalfa es una de las más usadas para la reducción de iones de plata, donde la caracterización por TEM muestra una variedad de formas de las nanopartículas a través de esta biosíntesis, también en Capsicum annuum se reporta la formación de las estructuras metálicas con un tamaño de $20 \mathrm{~nm}$, en un tiempo de reacción de 9hrs y de $40 \mathrm{~nm}$ en $13 \mathrm{hrs}$, la forma que se presenta es esférica. (Romero, 2014). Ledezma et al., sintetizaron nanopartículas de plata (AgNPs), utilizando extracto acuoso de nopal como agente bioreductor en presencia de polímeros biocompatibles solubles en agua poli (vinil alcohol) [PVA] como agente de estabilización de las nanopartículas. (Baylón, 2015).

Las Prosoplis juliflora Sw (mezquite) y Pluchea 74erícea Nutt (cachanilla) son plantas con la capacidad de generar metabolitos secundarios biológicamente activos susceptibles de convertirse en fitonanopartículas (fitoNPs) metálicas que aumentan su potencia y espectro biológico, ya que los compuestos presentes en los extractos acuosos del mezquite y cachanilla son capaces de transformarse en fitonapartículas estables de cobre y zinc, empleando técnicas de síntesis verde utilizando disoluciones de $\mathrm{CuSO}_{4}$ y $\mathrm{ZnSO}_{4}$ respectivamente, las fitoNPs de mezquite presentan un tamaño de partículas metálicas de $\mathrm{Cu}$ y $\mathrm{Zn}$ de 33.8 y $51.25 \mathrm{~nm}$ respectivamente vs 68 y $53.4 \mathrm{~nm}$ las de cachanilla, presentando una formas elipsoidales y esféricas de las fitoNPs en presencia de cobre y formas irregulares por la interacción del Zn, el estudio realizado de la actividad biológica demostró las fitoNPs-Zn presentaron mayor efecto insecticida, incluyendo daño celular y mortalidad sobre Phenacoccus solenopsis, seguidas de las fitoNPs$\mathrm{Cu}$ de esta planta, pero sin mortalidad. 
Dichos resultados alientan a continuar explorando las propiedades biológicas y químicas de fitonanopartículas botánicas de estas y otras especies nativas (Jiménez, 2020).

Como se muestra todos estos procesos biológicos son limpios en comparación con procesos químicos que necesitan emplear sustancias tóxicas y peligrosas, mientras que con la biosíntesis podemos encontrar el mismo resultado al utilizar agentes biológicos a temperatura ambiente.

Por tanto, la biosíntesis es una excelente respuesta para la incursión en nanotecnologías; y siguiendo con la línea de investigación en el presente trabajo se utilizó el lirio acuático (Echhornia Crassipes) para la biosíntesis de nanometales en residuos de pilas zinc- carbón.

\subsection{Lirio Acuático}

Nombre científico: (Eichhornia Crassipes) es una planta hidrófita libre flotadora perteneciente a la familia de las Pontederiaceae (Malik, 2007), Originaria del Amazonas en Sudamérica (Lindsey et col., 2000). Presenta una morfología como planta flotadora, perene, que posee hojas de color verde brillante, espigas florales de color violeta o amarillas y una raíz fibrosa que puede llegar a medir hasta $3 \mathrm{~m}$ de largo (Lindsey y Hirt, 2000; Gakwavu, 2007).

Sin embargo, resulta la E. Crassipes se ha convertido en un problema en diversas partes del mundo (Malik, 2007), empeorando las condiciones de vida de la gente que vive en los alrededores de los cuerpos de agua invadidos, ya que las matas que flotan sobre el agua, al ser arrastradas por el viento pueden servir como vectores de transporte de los organismos causantes de enfermedades como la malaria demás de volverse sitios de riesgo debido a animales como; serpientes y otros animales venenosos (Gunnarsson et col., 2007).

Debido a la mano del hombre, el lirio acuático se ha dispersado por todas las zonas tropicales del mundo (Gunnarsson et col., 2007) lo que ha provocado que actualmente se le considere como maleza nociva y esté atrayendo la atención global debido a que estas características provocan un fuerte impacto al ambiente, a la salud humana y al desarrollo económico (Malik, 2007). Pese a que el lirio pareciera ser el responsable de todos los problemas mencionados anteriormente, se debe de pensar positivamente en esta planta como una fuente de biomasa y proteína para muchos procesos actuales. Por su rápido crecimiento, que es una característica muy apreciada en cualquier cultivo, y su capacidad de remover contaminantes de los cuerpos acuíferos, la E. Crassipes puede ser considerada como una materia prima potencial para diferentes industrias como: la de los alimentos funcionales, la de biocombustibles (etanol y diésel) o en investigación para bio y fito - remediación (Gunnarsson et col., 2007; Malik, 2007).

Un documento importante en relación con el lirio acuático es un estudio de evaluación de remoción de indicadores fecales, donde se demostró que en aguas contaminadas por materia fecal sometidas a la presencia de la E. Crassipes, disminuyo hasta un $99 \%$ de la presencia de colíformes fecales. También es sabido que la E. Crassipes, es una de las plantas acuáticas con un gran potencial en la fitorremedación de metales pesados debido a su tolerancia y alta capacidad para acumularlos. Investigaciones documentadas han demostrado que la E. Crassipes puede ser útil para el tratamiento de aguas contaminadas con $\mathrm{As}, \mathrm{Be}, \mathrm{Cd}, \mathrm{Cr}, \mathrm{Hg}, \mathrm{Ni}, \mathrm{Pb}, \mathrm{Se}$ y Zn.

Los estudios ya realizados en los lirios de fito y biorremediación estos son capaces de acumular los metales pesados y la presencia de éstos disminuyen de la siguiente manera: raíz> tallo> hojas> frutos> semillas (Blum, 1997).

Las pilas desde su descubrimiento a inicios del siglo XIX por el científico Alessandro Volta, que determino que existía un flujo continuo de fuerza eléctrica al usar ciertos líquidos como conductores, a través de una serie de discos metálicos, promoviendo así una reacción química que genera energía. (Ortiz et col., 2008). Estas han tenido grandes aplicaciones y modificaciones con el paso de los años ya que una pila eléctrica es un dispositivo capaz de convertir la energía química en eléctrica por medio de una reacción de Oxidación-Reducción, y que, gracias a este descubrimiento, se ha llegado a obtener pilas de todo tipo y tamaño. 
La duración, voltaje y clasificación de una pila depende de los componentes de esta, entre los componentes más comunes se encuentran metales como, Cadmio (Cd), Litio (Li), Manganeso (Mn), Mercurio $(\mathrm{Hg})$, Níquel $(\mathrm{Ni})$, Plata $(\mathrm{Ag})$, Plomo $(\mathrm{Pb})$ y Zinc $(\mathrm{Zn})$ incluyendo un medio conductor de corriente eléctrica, que puede ser un electrodo de carbón o un electrolito a base de hidróxido (potasio o sodio) y cloruro de sodio, entre otros (AMEXPILAS, 2008).

Sin embargo, el uso indiscriminado de las pilas y baterías ha ocasionado una gran problemática a nivel ambiental y de salud por su alto nivel de contaminación en suelos, agua y aire, lo que ocasiona daños irreversibles al ecosistema, al igual que en la salud humana ya que algunos componentes de las pilas pueden ocasionar daños en el sistema respiratorio, riñones, cáncer de todo tipo, etc.

Por lo antes mencionado, en esta investigación se utilizó la biomasa del Lirio acuático (Eichhornia Crassipes) como agente biorreductor, con la finalidad de obtener un método ecológico y económico en la obtención de nanopartículas de $\mathrm{ZnO}$ y $\mathrm{MnO}$ utilizando pilas descartadas de Zinc-carbón tipo (AA) ya que existen pocos métodos de recuperación de residuos de pilas que sean ecológicos y que brinden un paso importante hacia la síntesis de éstas.

\subsection{Metodología}

Para esta investigación se utilizaron dos tipos de pilas alcalinas (AA) ya descartadas, las cuales fueron Sony Polad (SP1) y Pleomax (PLx), durante la recolección de pilas para la realización de la biosíntesis de nanopartículas se donaron una gran cantidad de pilas de diferentes marcas algunas ya se encontraban con un avance de deterioro grave estaban hinchadas, semi-abiertas, se encontraban oxidadas, etc; se eligieron las pilas antes mencionadas por ser estás la que más demanda de adquisición presenta en la población del Oriente del Estado de México donde se realizó la colecta.

Para realizar la biosíntesis de $\mathrm{ZnO}$ y MnO de las pilas alcalinas (AA) Sony Polad (SP1) y Pleomax (PLx) se realizaron los siguientes pasos:

\subsection{Extracción de muestra}

Se emplearon unas pinzas especiales para cortar carcasa de las pilas, abriéndolas por la mitad con mucho cuidado para no dañar el ánodo y poder separar manualmente todos los componentes y recuperar la pasta interna donde se concentran todos los componentes químicos.

Posteriormente se separó el electrolito (pasta negra) y se dejó secar durante 24 horas a temperatura ambiente de $25^{\circ} \mathrm{C}$, transcurrido ese tiempo se trituro en un mortero de ágata hasta obtener un material fino. Una vez obtenido el material se procedió a preparar las muestras colocándolas durante 36 horas en la mufla a $900^{\circ} \mathrm{C}$ con la finalidad de obtener fases bien definidas en los cristales característicos del electrolito.

Para la identificación de las muestras obtenidas se realizaron estudios espectroscópicos como difracción de Rayos X, TEM-EDX y TEM utilizando un microscopio HIRTEM y HRSEM con espectroscopia EDS. Para ello, se prepararon los lirios acuáticos de la siguiente manera

\subsection{Preparación del lirio acuático (Eichhoirnia Crassipies)}

a. Lavado con agua y detergente. El lirio fue lavado con la finalidad de quitar cualquier residuo de lodo con agua común y detergente. Posteriormente, los lirios fueron cortados en pequeños trozos para su facilitar el secado en el horno a $80 \square 5^{\circ} \mathrm{C}$ durante una semana.

\section{b. Lavado del lirio con solución ácida}

Una vez seco el lirio acuático se trituró en un mortero de ágata y se agregó $\mathrm{HCl} 0.01 \mathrm{M}$ a una relación 3g: $100 \mathrm{~mL}$, se agitó y se dejó en reposo durante 30 minutos. Finalmente, se filtró el lirio con una tela de algodón, recuperando el filtrado (lixiviado) y la biomasa la cual se lavó con agua desionizada a la misma relación sólido: líquido para remover el exceso de ácido y nuevamente se procedió a filtrar la mezcla para recuperar la biomasa y secarla a $80 \square 5^{\circ} \mathrm{C}$ durante una semana 


\subsection{Biosíntesis de nanopartículas metálicas}

Se efectuó la síntesis de nanopartículas de Mn, del orden de 1 a 20 nm, por el método de bioreducción utilizando la biomasa de los lirios. Para determinar las condiciones óptimas de biosíntesis se efectuaron variaciones en cuanto a las variables de procesamiento del lirio acuático: ajuste de $\mathrm{pH}$ el cual se controló por medio de una solución de tampón a los valores de 3, 5, y 10, concentración de la suspensión de la biomasa, concentración de la solución metálica, principalmente. A continuación, se detalla el método implementado:

\subsection{Preparación de nanopartículas}

- Se prepara una solución del metal en estudio con agua des ionizada y se somete a baño ultrasónico $20 \mathrm{~min}$.

- Se preparó con una concentración de $5 \mathrm{mg}$ de lirio/mL en agua destilada usando un tratamiento de ultrasonidos durante $15 \mathrm{~min}$, la cual es vaciada en cada tubo de ensaye.

- Posteriormente, las muestras se centrifugan a $5000 \mathrm{rpm} 15 \mathrm{~min}$

- A cada muestra se le agrega $1 \mathrm{~mL}$ de solución buffer en la solución de biomasa.

- Después se coloca la mezcla en un baño de ultrasonidos durante 15 min, Y se deja durante otros 10 minutos sin agitación y después se centrifuga a 5000 rpm durante 15 min.

- A continuación, a cada muestra se le adiciona $1 \mathrm{~mL}$ de solución metálica/mL de solución de biomasa.

- Posteriormente, la solución mezcla se somete a baño ultrasónico durante 20 minutos y, una vez concluido, se centrifugan a $5000 \mathrm{rpm} / 20 \mathrm{~min}$ a $25^{\circ} \mathrm{C}$.

- Teniendo cuidado de no mover las muestras, se separa el sobrenadante (solución coloidal) del precipitado (biomasa) por decantación, este último es desechado y la solución es analizada por Microscopía Electrónica de Transmisión (TEM).

\subsection{Resultados y discusiones}

\subsection{Estudio de Difracción de DRX}

Una vez obtenido los electrolitos de las pilas Zinc-Carbón Sony Polad (SP1) y Pleomax (PLx), se realizó un estudio de difracción de Rayos X con la finalidad de identificar la composición elemental de los metales tóxicos presentes en las pilas analizadas, las cuales fueron secadas previamente en la mufla en tiempos de 0 a 36 horas a temperatura ambiente de $25^{\circ} \mathrm{C}$ y $900^{\circ} \mathrm{C}$, en la figura 6.1 , se muestran los espectros de DRX. 
Figura 6.1 Espectros de difracción de Rayos X, obtenidos en la pila Sony Polad (SP1) a diferentes: a) $\mathrm{T}=25^{\circ} \mathrm{C}$ y un $\mathrm{t}=10 \mathrm{hrs}$; b) $\mathrm{T}=900^{\circ} \mathrm{C}$ y $\mathrm{t}=36 \mathrm{hrs}$
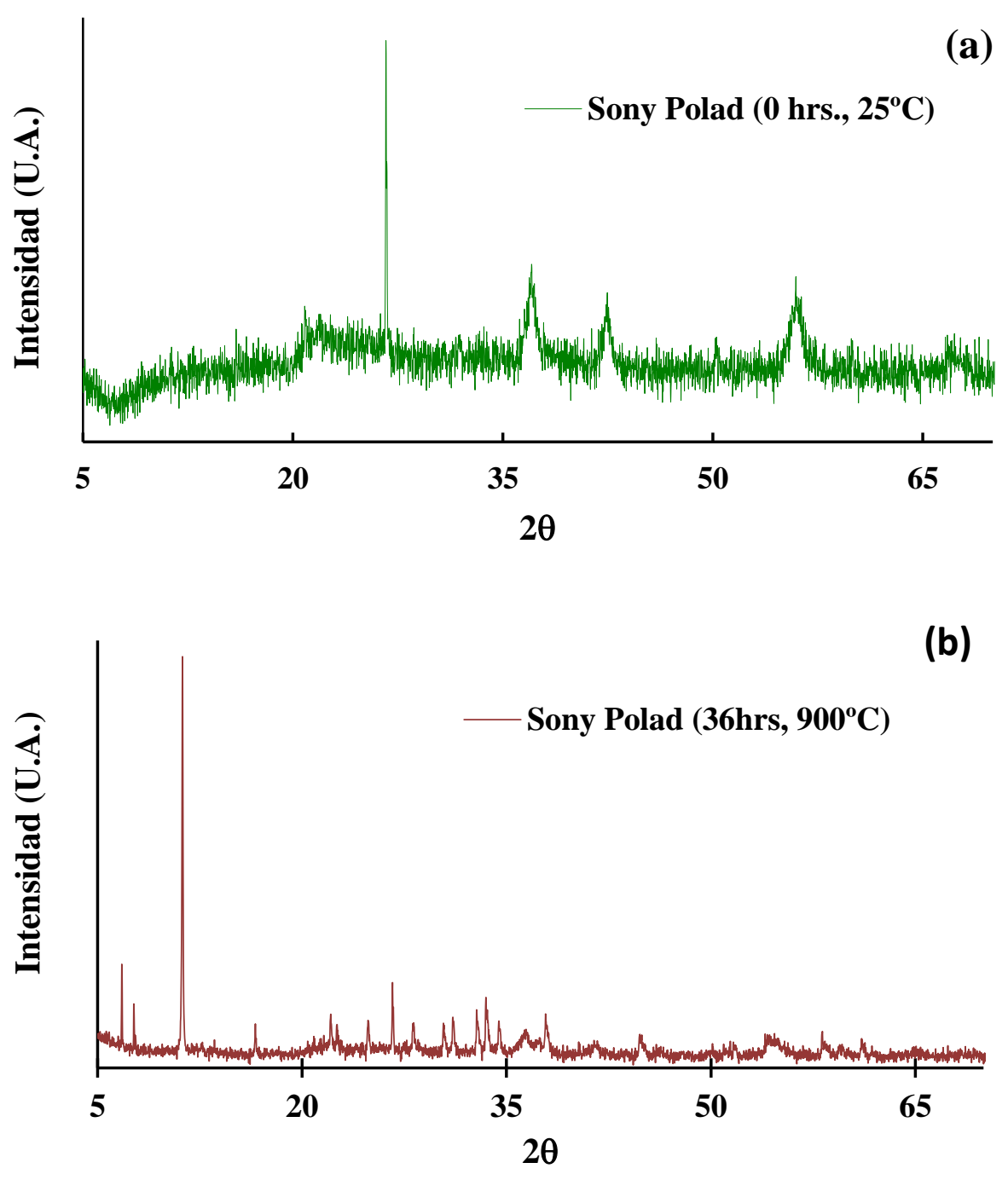

En la figura 6.1, Se observa los cambios de cristalización en las dos temperaturas empleadas y los tiempos asignados, es importante resaltar que se emplearon otras temperaturas y tiempos para la obtención de los EDX sólo se muestran los dos espectros donde se observan con mayor claridad los cambios de cristalización e identificación de las fases presentes, $\mathrm{MnO}$ y $\mathrm{ZnO}$ figura 1.1 a) $\mathrm{T}=25^{\circ} \mathrm{C}, \mathrm{t}=10$ horas y figura $1.1 \mathrm{~b}$ ) $\mathrm{T}=900^{\circ} \mathrm{C}, \mathrm{t}=36$ horas obtenidos de la muestra (SP1) en esta se define mejor la estructura cristalina.

En la figura 6.2, se muestran los espectros DRX de la pila Pleomax (PLx) en los cambios cristalinos en las mismas condiciones de análisis de la pila SP1. 
Figura 6.2 Espectros de difracción de Rayos X, obtenidos en la pila Pleomax (PLx) a diferentes: a) T= $25^{\circ} \mathrm{C}$ y un $\mathrm{t}=0 \mathrm{hrs}$; b) $\mathrm{T}=900{ }^{\circ} \mathrm{C}$ y $\mathrm{t}=36 \mathrm{hrs}$
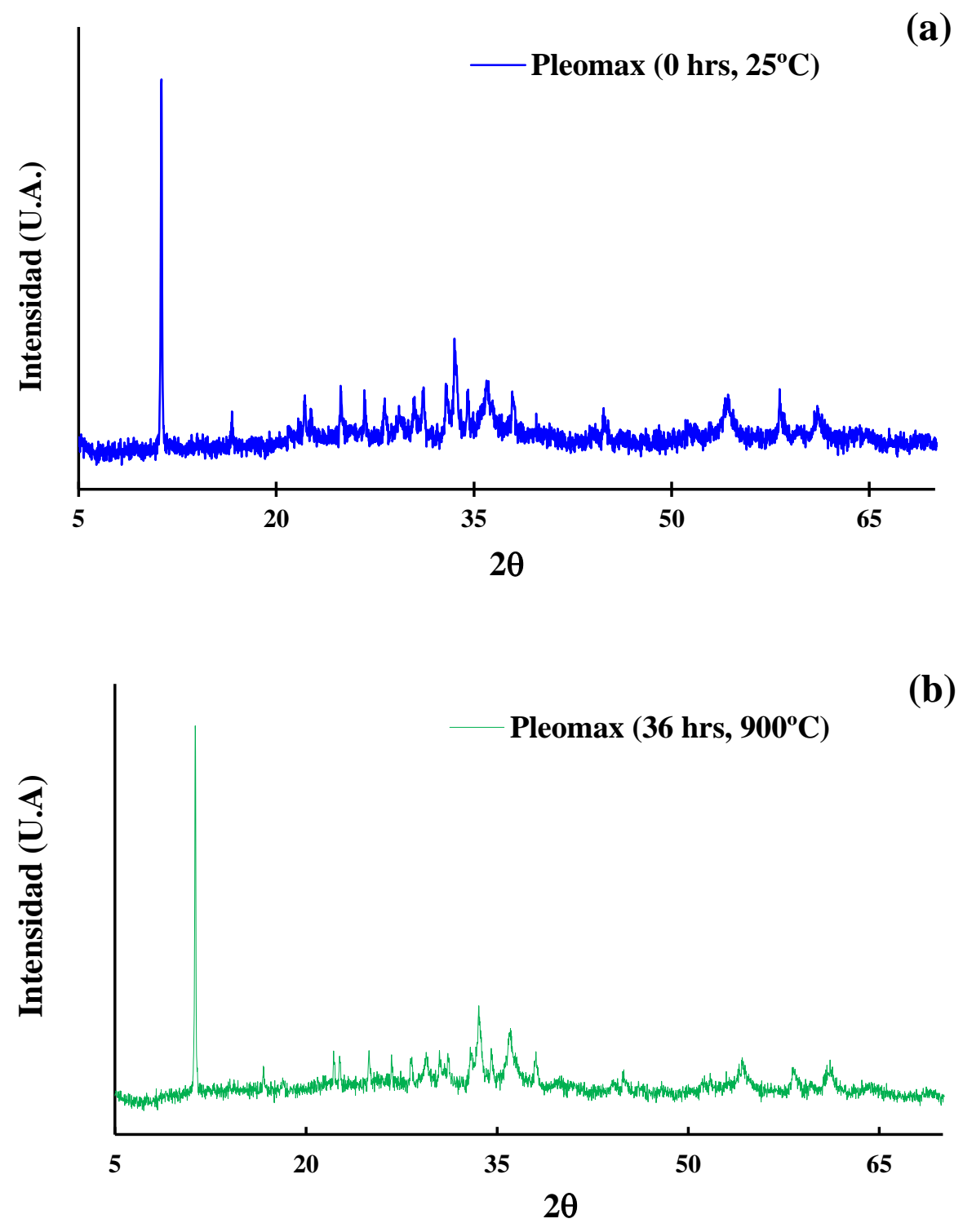

En la figura 6.2 a) y b), se observa que aún con los cambios de temperatura y tiempo, la cristalización es menor que en las muestras anteriores, es importante mencionar que las muestran presentaron un comportamiento higroscópico desde su extracción y aun cuando se secó a temperatura ambiente $25^{\circ} \mathrm{C}$ a 10 horas y en la mufla a $900^{\circ} \mathrm{C}$ durante 36 horas al momento de entra en contacto con el medioambiente se humedecía, impidiendo una tener una buena lectura en la cristalización.

En general, se observa que a $900^{\circ} \mathrm{C}$ y después de haber transcurrido 36 horas las muestran mejoran la cristalización, definiéndose las fases esperadas de $\mathrm{MnO}$ y $\mathrm{ZnO}$. Es importante mencionar que aun a esta temperatura las muestras no terminaron de cristalizar y definirse en su totalidad, ya que en cada intervalo de tiempos se observaban más señales, por su comportamiento higroscópico fue difícil tener una caracterización con el software del equipo, por lo que se realizó un estudio de Microscopia Electrónica a Trasmisión con microanálisis (TEM-EDS) con la finalidad de caracterizar los compuestos presentes en las muestras sometidas a $900^{\circ} \mathrm{C}$ y 36 horas.

\subsection{Estudio de Microscopia electrónica a Trasmisión con microanálisis TEM- EDS}

Se realizó un estudio de TEM-EDS a las muestras del electrolito de las pilas Zinc-carbón (SP1 y PLx) las cuales fueron sometidas a una $\mathrm{T}=900^{\circ} \mathrm{C}$ y $\mathrm{t}=36$ horas. En la figura 3 , Se muestran los espectros de los componentes químicos de la pila descartada Sony Polad (SP1), los cuales fueron obtenidos mediante la técnica TEM-EDS. 
Figura 6.3 SEM-EDS de la muestra (SP1)

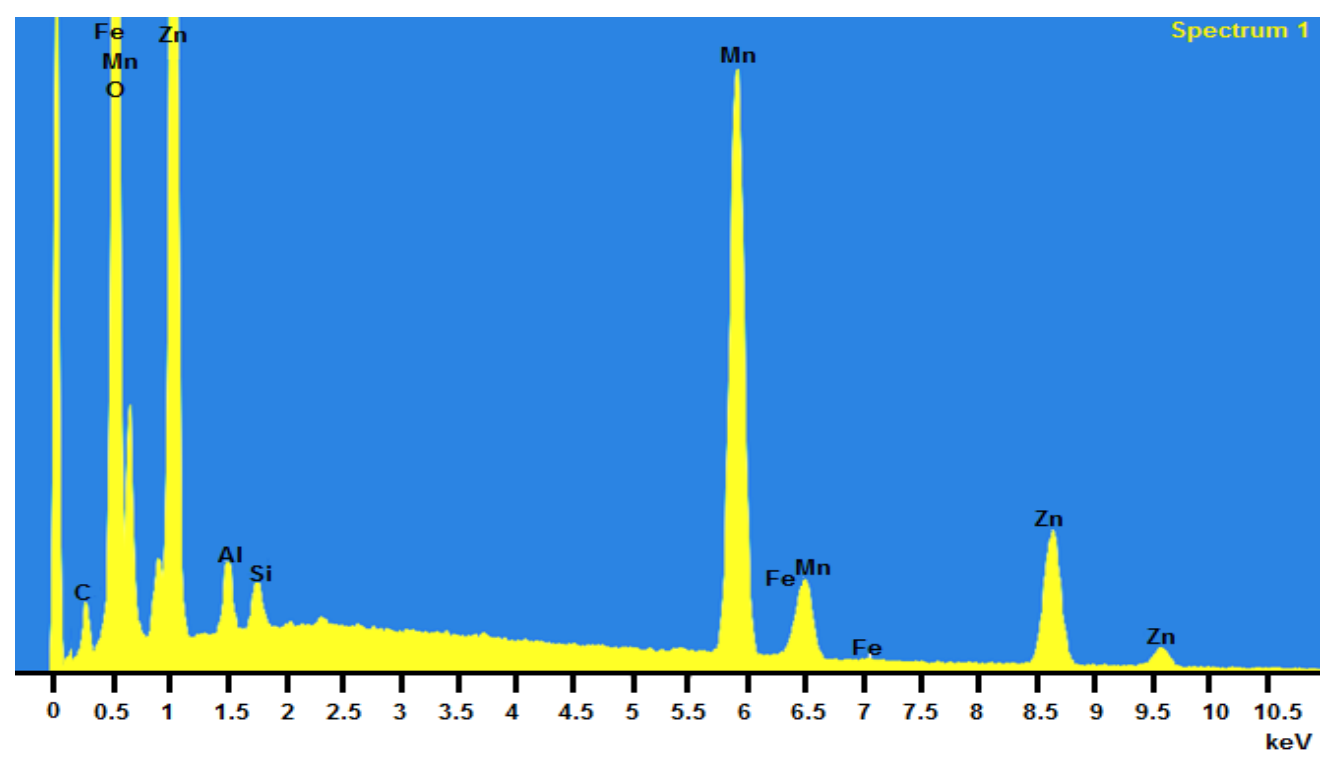

En la Figura 6.3, se observa que los óxidos de Manganeso MnO están muy por encima de los límites permisibles, existe la presencia de Silicio ( $\mathrm{Si}$ ) que no es un componente común en las pilas la presencia de aluminio $\mathrm{Al}$ ) y Hierro $(\mathrm{Fe})$ se asume que se debe al material de la carcasa de éstas, dichos análisis corroboran que no cumplen con los estándares de calidad establecidos en la elaboración de éstas.

En la tabla 6.1, se muestran los resultados obtenidos de los componentes químicos presentes en la muestra Sony Polad (SP1), donde se observa la presencia de óxidos de manganeso (MnO), Zinc (Zn), Carbono (C), Silicio (Si), Oxígeno (O), etc.

Tabla 6.1 Composición Química de la pasta de la muestra (SP1)

\begin{tabular}{|l|r|r|r|}
\hline Elemento & \multicolumn{1}{|c|}{ Peso\% } & \multicolumn{1}{c|}{ Peso\% } & \multicolumn{1}{l|}{ Atómico\% } \\
\hline $\mathbf{C}$ & & \multicolumn{1}{|c|}{ Sigma } & 8.22 \\
\hline $\mathbf{O}$ & 3.16 & 0.15 & 54.95 \\
\hline $\mathbf{A l}$ & 28.16 & 0.14 & 1.06 \\
\hline $\mathbf{S i}$ & 0.92 & 0.03 & 0.64 \\
\hline $\mathbf{M n}$ & 0.58 & 0.03 & 18.46 \\
\hline $\mathbf{F e}$ & 32.48 & 0.15 & 0.66 \\
\hline $\mathbf{Z n}$ & 1.19 & 0.10 & 16.01 \\
\hline Totales & 33.52 & 0.16 & \\
\hline
\end{tabular}

En la Figura 6.4, Se muestran los espectros de los componentes químicos de la pila descartada Pleomax (PLx) analizados mediante la técnica TEM-EDS. 
Figura 6.4 TEM de la muestra (PLx)

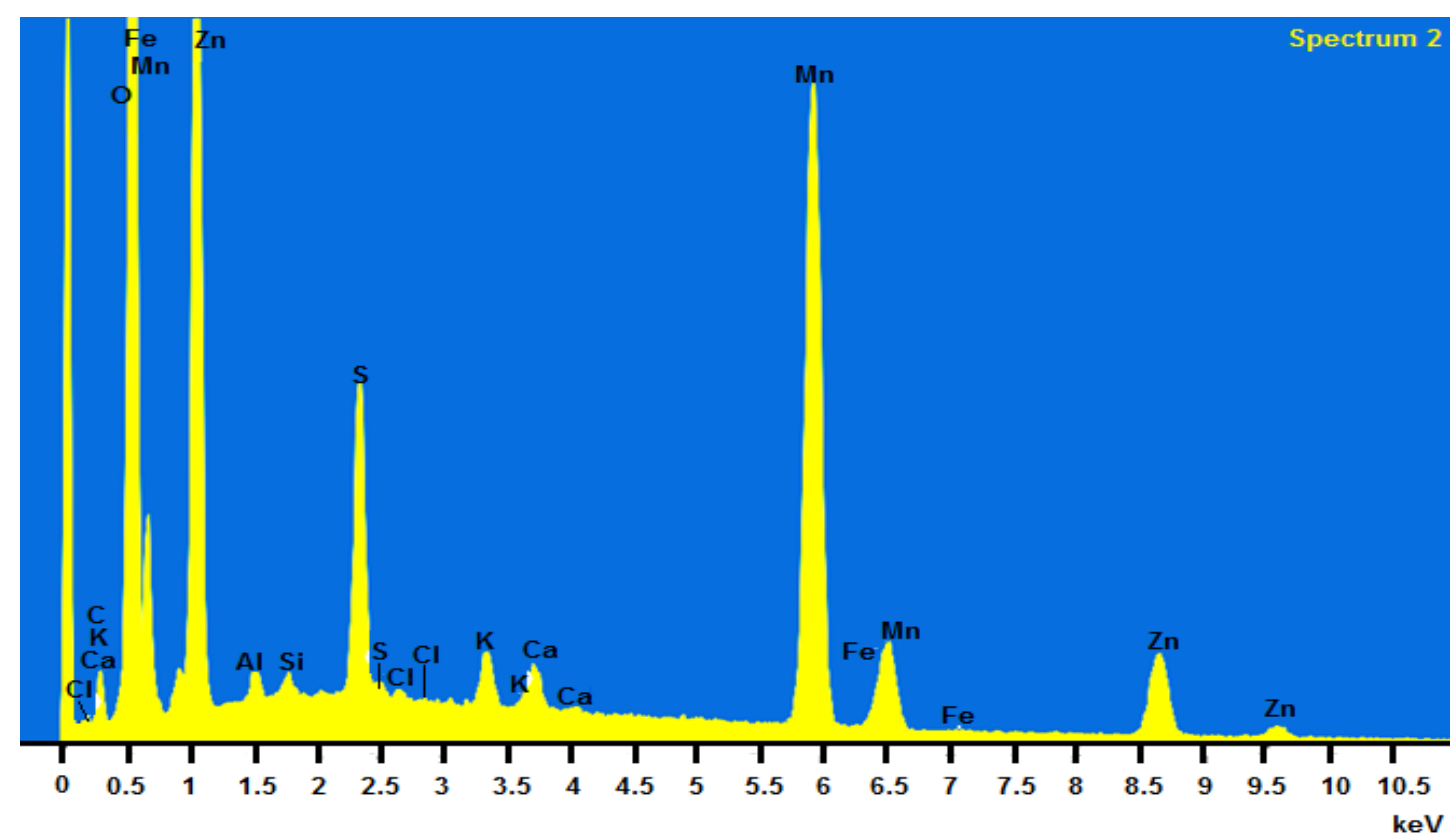

En la Figura. 6.4, Se puede observar que predomina una cantidad mayor de $\mathrm{MnO}$ y $\mathrm{ZnO}$ que son los componentes principales de la reacción de oxidación de las pilas Zinc-carbón, se observa de nuevo la presencia de $\mathrm{Si}$ y otros componentes no comunes en las pilas. Los resultados obtenidos de la muestra PLx en los componentes de Manganeso (Mn) están por encima de lo reportado por la Eurotech 1991 y al RIS Internacional 2007, los demás elementos se encuentran por debajo de lo establecido.

En la Tabla 6.2, se muestran los resultados obtenidos de los componentes químicos presentes en la muestra Pleomax (PLx).

Tabla 6.2 Composición Química de la pasta de la muestra (PLx)

\begin{tabular}{|l|l|l|l|}
\hline Elemento & Peso\% & Peso\% & Atómico\% \\
\hline $\mathbf{C}$ & & Sigma & \\
\hline $\mathbf{O}$ & 3.11 & 0.24 & 7.54 \\
\hline $\mathbf{A l}$ & 31.55 & 0.17 & 57.50 \\
\hline $\mathbf{S i}$ & 0.36 & 0.03 & 0.39 \\
\hline $\mathbf{S}$ & 0.21 & 0.03 & 0.22 \\
\hline $\mathbf{C l}$ & 3.93 & 0.05 & 3.58 \\
\hline $\mathbf{K}$ & 0.16 & 0.03 & 0.13 \\
\hline $\mathbf{C a}$ & 0.92 & 0.03 & 0.68 \\
\hline $\mathbf{M n}$ & 0.77 & 0.03 & 0.56 \\
\hline Fe & 35.41 & 0.18 & 18.79 \\
\hline $\mathbf{Z n}$ & 1.07 & 0.10 & 0.56 \\
\hline Total & 22.51 & 0.17 & 10.04 \\
\hline & $\mathbf{1 0 0 . 0 0}$ & & \\
\hline
\end{tabular}

En la tabla 6.2, Se observa que en la muestra PLX existe la presencia de compuestos químicos que no son muy comunes en otras pilas, tales como el Azufre (S), Cloruros $(\mathrm{Cl})$ y el Silicio $(\mathrm{Si})$, lo que nos confirma que existen pilas en el mercado que no cumplen con las medidas establecidas y no tienen un control de calidad.

A continuación, se muestran los resultados de Microscopia Electrónica de Transmisión TEM de las muestras de la biosíntesis de nanometales utilizando el lirio acuático como agente biorreductor.

\subsection{Resultados de la Biosíntesis de nanometales, empleando la técnica de Microscopia Electrónica de Transmisión (TEM)}

Con la finalidad de caracterizar (estructura y distribución de tamaño) las nanopartículas de Mn y Zn sintetizadas por el método de biorreducción utilizando la biomasa de lirio acuático, se empleó la técnica TEM utilizando un microscopio HRTEM y HRSEM con espectroscopia EDS de la UAM- Iztapalapa, con este fin se extendió la muestra en papel filtro Whatman grado 3 y se dejó secar al ambiente. 
En la figura 6.5, se muestran el mapeo obtenido mediante TEM de la muestra SP1 a un valor de $\mathrm{pH}=3$ establecido para la bioreducción, a una escala de: a) $5 \mathrm{~nm}$ y b) $10 \mathrm{~nm}$.

Figura 6.5 a) $\mathrm{pH}=3$, Escala: $5 \mathrm{~nm} \quad$ Imagen 1.1. b) $\mathrm{pH}=3$, Escala: $10 \mathrm{~nm}$
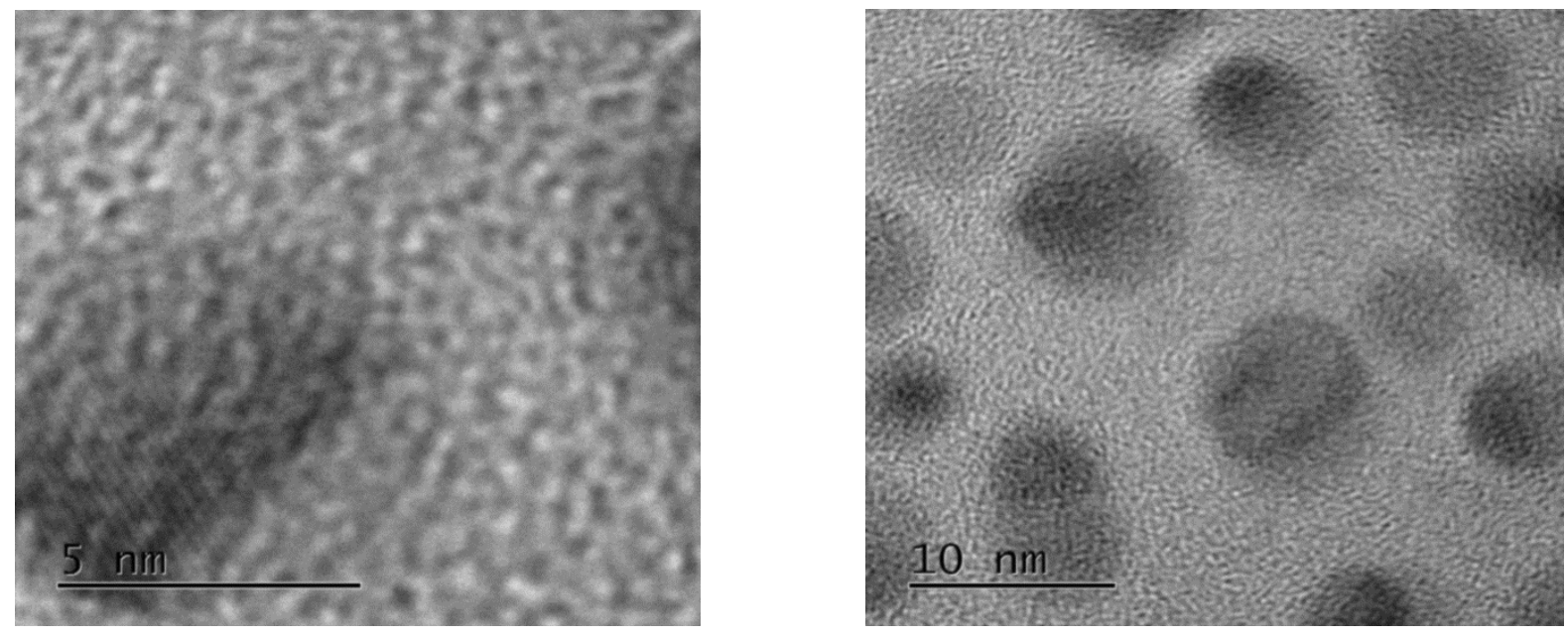

En la Figura 6.5 se observa que en: 1.1a) en la escala de 5nm, se observan varias nanopartículas con una buena orientación en los planos cristalográficos, mientras que en 1.1b) escala de 10nm se aprecia una buena definición y distribución de las nanopartículas de Mn y Zn, así como planos cristalográficos bien definidos.

En la imagen 6.6, se muestran la imagen obtenida del mapeo realizado mediante TEM de la muestra PLx a un valor de $\mathrm{pH}=3$ establecido para la bioreducción, a una escala de: a) $5 \mathrm{~nm}$ y b) $10 \mathrm{~nm}$.

Figura 6.6 pH=3, Escala: $5 \mathrm{~nm}$

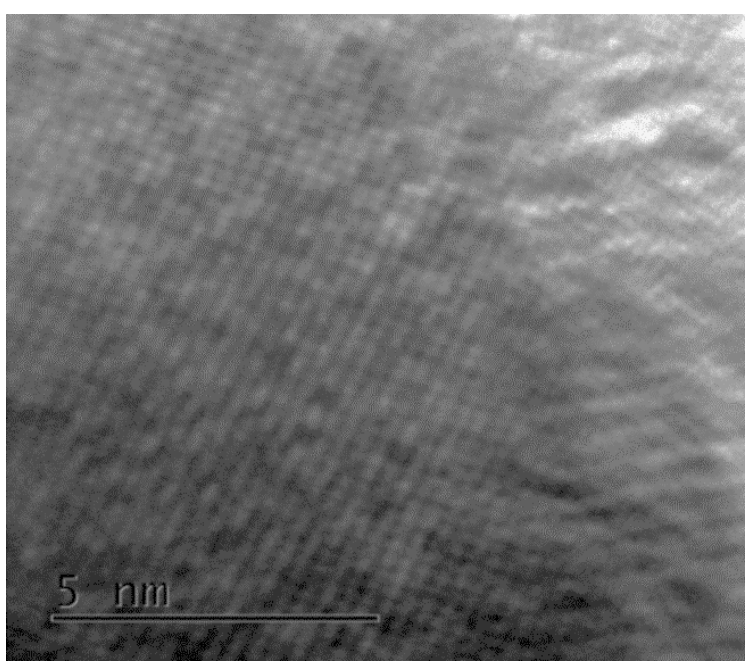

Figura 6.6 pH=3, Escala: $10 \mathrm{~nm}$

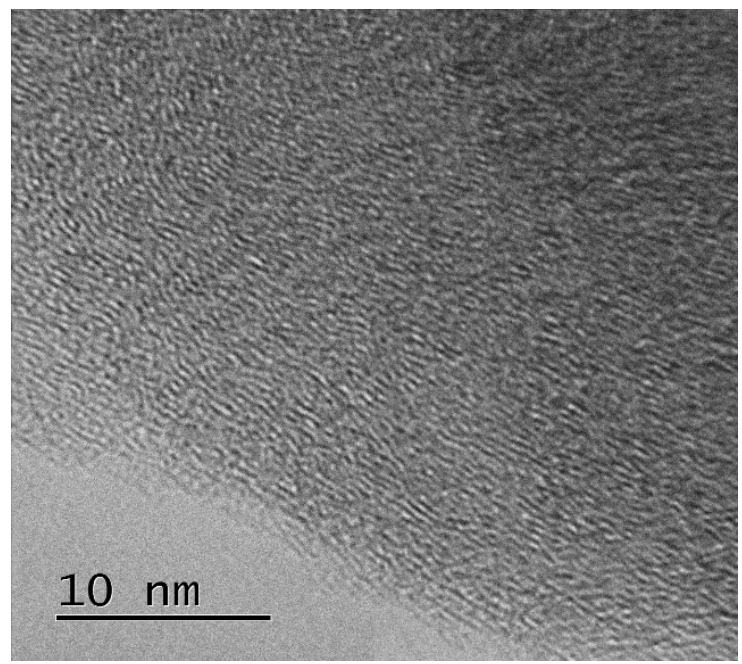

En la figura 6.6, se observa que en: 1.2a) en la escala de 5nm, y 1.2b) escala de 10nm, en ambas se aprecia una buena definición y distribución de las nanopartículas de Mn y Zn, así como planos cristalográficos bien definidos, y con un tamaño uniforme de las nanopartículas. Se observa la aglomeración de las nanopartículas y se aprecia una formación de cúmulos.

\subsection{Agradecimientos}

Al Tecnológico de Estudios Superiores del Oriente del Estado de México por brindar todas las facilidades para llevar a cabo esta investigación.

A la Universidad Autónoma Metropolitana por permitir que se llevará a cabo esta investigación en sus instalaciones en el Dpto. de Química área Electroquímica y Dpto. área de Ingeniería en Recursos Energéticos. 


\subsection{Conclusiones}

Se obtuvieron reproducibles muestras de manera satisfactorias las nonopartículas metálicas de $\mathrm{ZnO}$ y $\mathrm{MnO}$ de las pilas descartadas de marca Sony Polad y Pleomax, empleando como bioreductor el lirio acuático.

Los estudios de difracción de Rayos X permitieron comprobar la presencia de las fases principales esperadas, $\mathrm{MgO}$ y $\mathrm{ZnO}$ y que se tiene una mejor cristalización de los componentes de las pilas empleadas cuando fueron sometidas a una temperatura de $900^{\circ} \mathrm{C}$ en un tiempo de 36 horas ya que muestran una mejor definición en las señales y disminución del ruido.

Mediante TEM fue posible identificar la composición de las pilas empleadas y se pudo observar que éstas muestran elementos que aún no son reportados en la literatura, lo que es claro que algunos fabricantes emplean catalizadores con la finalidad de tener un mejor rendimiento, sin considerar que al ser descartadas las pilas terminan en los basureros a cielo abierto y reaccionan los componentes, contaminando de una manera desmedida e irreversible el suelo, agua, animales, etc.

Es muy importante resaltar que en los resultados obtenidos en las dos muestras de pilas analizadas muestran límites superiores de los permisibles en los componentes superiores a los reportados por la Unión Europea. Aún falta mucho por realizar en relación con este trabajo ya que existen una gran variedad de marcas de pilas y en esta investigación solo se analizaron dos marcas ya antes mencionadas, es importante tomar conciencia sobre la disposición final de las pilas ya que están presentan límites de sus componentes por arriba de los permisibles en $\mathrm{MnO}$ y $\mathrm{ZnO}$, sin cuantificar aún a detalle los componentes nuevos encontrados; por ello, es urgente que se establezca nuevas políticas de disposición final y se creen Normas Mexicanas para la disposición final de este tipos de residuos tóxicos.

\subsection{Referencias}

Amexpilas, (2018), Estudios http://www.amexpilas.org/amexpilashtml/estudios.htmlRealizados.

Ara, B. N., Mondal, S. Basu, S. Laskar, R.A. Mandal, D. (2009), Biogenic synthesis of Au and Ag nanoparticles using aqueos solutions of black Tea leaf extracts Colloids and Surfaces B: Biointerfases. 71: $113-118$

Ascencio Jorge A, Rodríguez-Monroy Ana C., Liu Hong Bo y Canizal Gerardo, (2004), Chemestry Letters, vol.33, No. 8

Baylon Palomino Maria Brenda Olivia, (2015), TESIS, Biosintesis de nanoparticulas Metalicas Utilizando Extracto Acuoso de Opuntia spp de Poblaciones silvestre y Cultivada. UNIVERSIDAD AUTONOMA AGRARIA ANTONIO NARRO, Saltillo Coahuila, agosto.

Blum, W.H., (1997), Cadmium uptake by higher plants. Proceedings of extended abstracts from the fourth international conference on the biogeochemistry of trace elements. Berkeley, USA: University of California, pp. 109-110.

Boldyrev, V. V. (2006). Mechanochemistry and mechanical activation of soliods. Russian Chemical Reviews, 75:177-189.

Emil Roudner, (2006) Chem. Soc. Rev., 35, 583.

Ershov, G. G. (1997). Metal ions in unusual and unstableoxidation sates in aqueus solutions: Preparation and properties. Russian Chemical Reviews. 66:93-106.

Faraday. M. (1957). Experimental Relations of gold (and other Metals) to light Philosophical Transactions of the royal Society Londres.

Faraday, M., (1875), Phil. Trans. R. Soc., 147, 145 
Frederix, F., J.M. Friedt, K. H. Choi, W. Laureyn, A. Campitelli, D. Mondelaers, G. Maes, G. Borghs,. (2003). Biosensing based on light absorption of nanoscaled gold and silver particles Analytical Chemistry. 75(24):6894-6900.

Gakwavu R. J. (2007). Zinc and chromium removal mechanisms from industrial wastewater by using water hyacinth, Eichhornia crassipes (mart.) Solms. Lambert Academic Publishing. pp 1-84.

Gaviria Restrepo, J., Pavón Juan José, Abdul Villarraga Junes, Segura-Sánchez Freimar, TorresHernández Yadir, Rodríguez José Antonio, (2019). Síntesis e inmovilización de nanopartículas de plata sobre substratos de titanio poroso con potencial uso en materiales implantables. Revista médica Universidad de Antioquia, IX SeminarioCiencias Básicas Biomédicas Latreria, vol. 32, pág. S-15

Gunnarsson C. C. y Mattsson P. C. (2007), Water hyacinths as a resource in agriculture and energy production: A literature review. Waste Management, 27, 117 - 129.

Jacquelyne Zarria-Romero, Ana Osorio, José Pino, Betty Shiga, Dan Vivas-Ruíz. (2017). Efecto de las Nanopartículas Industriales $\mathrm{TiO}_{2}, \mathrm{SiO}_{2}$ y $\mathrm{ZnO}$ sobre la viabilidad celular y expresión génica en médula ósea roja de Mus musculus. Rev Peru Med Exp SaludPublica, 34 (3), 436-444

Jiménez León E. del Carmen. (2020). Tesis "Fitonanopartículas de plantas nativas del Valle de Mexicali: Caracterización y evaluación de sus propiedades antimicrobianas e insecticidas". Universidad de Ciencias y Artes de Chiapas- Instituto de Ciencias Biológicas, 1-89.

Karuvath Yossaf, Binil Itty Ipe, Cherumuttathu H. Suresh, and K. George Thomas J. (2007), Phys. Chem. $111,12839$.

Lindsey K. y Hirt H. M. (2000), “ ¡Usos del lirio acuático!! Un manual práctico para usos del lirio acuático alrededor del mundo". Anamed International. pp 2 -4, 5 -8, 21-30 y 31-32.

Malik A. (2007). Environmental challenge vis a vis opportunity: The case of water hyacinth. Environment International, 33, $122-138$.

Muzafarov. A. M. Rebrov, E. A. (2000), Current Trends in the Chemistry of Dendrimers, Polymer Science. 42: 55-77.

Taniguchi N., Proc. Intl. Conf. Prod. Eng., Tokyo, Part II, 1974

Ortiz Sandoval Ana Elena, Karen Rocío Parada Arévalo, (2018), "propuesta para la gestión ambiental de pilas y baterías (dispositivos electroquímicos generadores de energía) fuera de uso en el salvador".

P.S. Schabes-Retchkiman, G. Canizal, R. Herrera-Becerra, C. Zorrilla, H.B. Liu, J.A.

Ascencio, (2006) Optical Materials 29, pp. 95.

Perdigón P., (2010), "Síntesis y caracterización de nanopartículas de Gd y Sm por el método de biorreducción”- Tesis de Licenciatura. UNAM. Facultad de Ingeniería.

Romero E. J. (2014), Comunicación personal. Departamento de Materiales Avanzados. Centro de investigación de Química Aplicada. Saltillo, Coahuila de Zaragoza, México.

R. Herrera-Becerra, C. Zorrilla, G. Canizal, P.S. Schabes- Retchkiman, H.B. Liu, L. Tavera Davila, G. Rosano-Ortega, L. Rendón y J.A. Ascencio, (2008), Journal of Nanoscience and Nanotechnology, Vol. 8,1 .

Serrano-Niño J.C., Contreras-Martínez C.A., Solís Pacheco J.R., Zamudio Ojeda A., Aguilar Uscanga B.R., Cavazos-Garduño A. (2020). Optimization of the biosynthesis of gold nanoparticle using Hypericum perforatum and evaluation of their antimicrobial activity. Revista Mexicana de Ingeniería Química, 19, No. 2, 889-902. 
Seung H. K., Yeonho C., Hwang D.J., Grigoropoulosa G., Jaewon Ch. Poulikakos D. P. (2006), Appl. Phys. Lett. 89, 141126

Wachs, I. E. (2001). Characterization of catalytic materials. Momentum Press. New York. United States of America.

Yu Y. Z., Qinhui X. B., (2004), Shape2Controlled Syntheses of Metal Nanoparticles, (Department of Chemistry, Tsinghua University, Beijing 100084, China).

Jun Y.W., Jang J.T., (2007) J. Chem., Adv. Exp. Med. Biol., 620, 85

Zhu, H, Zhang, C., Yin, Y. (2004). Rapid Synthesis of copper nanoparticles by sodium hypophosphite reduction in ethylene glicol under microwave irradiation. Journal of Crystal Growth. 270: 722-728 